\title{
Somatic mutations of calreticulin in myeloproliferative neoplasms
}

\author{
Misa Imai $^{1,2} \cdot$ Marito Araki $^{3} \cdot$ Norio Komatsu ${ }^{1}$
}

Received: 25 April 2017 / Accepted: 26 April 2017 / Published online: 3 May 2017

(C) The Japanese Society of Hematology 2017

\begin{abstract}
Recurrent somatic mutations in calreticulin $(C A L R)$ gene that encodes a molecular chaperone residing in the endoplasmic reticulum were identified in 2013 in a subset of patients with myeloproliferative neoplasms (MPNs). All of these mutations found in patients were either small insertion or deletion in a narrow region on exon 9 of $C A L R$ gene, and caused +1 frameshift in the reading frame for the translation of the carboxyl-terminus of CALR. Because of this unique feature, the CALR mutation is believed to be a gain-of-function mutation. However, there was essentially no rationale model to implicate the involvement of mutant CALR in the pathogenesis of MPN or other malignancies. Based on the recent findings, this review summarizes a novel molecular mechanism by which this mutant molecular chaperone constitutively activates the cytokine receptor to induce cellular transformation in MPNs.
\end{abstract}

Keywords Myeloproliferative neoplasms . Thrombopoietin receptor · Calreticulin · Autocrine . Chaperone $\cdot$ MPL

Misa Imai and Marito Araki contributed equally to this work.

Norio Komatsu

komatsun@juntendo.ac.jp

1 Department of Hematology, Juntendo University Graduate School of Medicine, 2-1-1 Hongo, Bunkyo-ku, Tokyo 113-8421, Japan

2 Leading Center for the Development and Research of Cancer Medicine, Juntendo University Graduate School of Medicine, Tokyo 113-8421, Japan

3 Department of Transfusion Medicine and Stem Cell Regulation, Juntendo University Graduate School of Medicine, Tokyo 113-8421, Japan

\section{Introduction}

Philadelphia chromosome-negative myeloproliferative neoplasms (MPNs) which include polycythemia vera (PV), essential thrombocytosis (ET), and primary myelofibrosis (PMF), are characterized by clonal proliferation of hematopoietic stem and progenitor cells, resulting in the increase in the number of mature cells of one or more blood cell lineages. After the identification of Janus kinase 2 (JAK2) and myeloproliferative leukemia protein $(M P L)$ mutations in patients with MPN in 2005 and 2006, respectively [1], these genetic alterations were recognized to constitutively activate cytokine receptors and cause tumorigenic transformation. These findings led to the use of JAK2 inhibitors for the treatment of patients with MPNs. However, approximately half of the patients with ET or PMF had unknown etiology as they were negative for JAK2 or MPL mutations. At the end of 2013, somatic mutations in the gene encoding calreticulin $(C A L R)$ were identified in the majority of patients with ET and PMF lacking JAK2 and MPL mutations $[2,3]$. CALR is a molecular chaperone residing in the endoplasmic reticulum (ER), and rational models implicating the involvement of CALR in the pathogenesis of ET or PMF were absent. Based on the recent findings, the molecular mechanism by which mutant CALR promotes MPN development will be described in this review.

\section{Domain structures of wild-type and mutant CALR}

CALR was first identified as a calcium-binding protein from the ER fraction of rabbit skeletal muscle in 1974 [4]. CALR is highly conserved among species and consists of three domains: (i) an amino (N)-terminal lectinbinding domain, which acts as a chaperone and contains a 
zinc-binding site, (ii) a proline-rich P-domain, containing high-affinity binding sites for $\mathrm{Ca}^{2+}$, and (iii) a carboxy (C)domain containing multiple low-affinity $\mathrm{Ca}^{2+}$-binding sites [5] (Fig. 1). The localization and retention of CALR are defined by the N-terminal signal sequence and the C-terminal ER-retention sequence KDEL. CALR is involved in a variety of intracellular (cytoplasm and nucleus), cell surface, and extracellular functions such as protein quality control, calcium metabolism, immune response, phagocytosis, cell adhesion, and so on [6].

In 2013, recurrent somatic mutations in $C A L R$ were identified in a majority of patients with ET and PMF who were negative for $J A K 2$ and $M P L$ mutations that are known as driver mutations for MPN. Mutations in CALR identified in those patients are exclusively frameshift mutations residing in exon 9 of the gene [2,3]. Although two mutations, a 52-base deletion (type 1: c.1092_1143del, hereafter called del52) and 5-base insertion (type 2: c.1154_1155insTTGTC, hereafter called ins5), account for approximately $80 \%$ of all CALR mutations, more than 50 types of CALR mutation have been reported so far. All of them are small insertions or deletions that cause $+2 /-1$ base-pair frameshift generating a mutant-specific 36 amino acid C-terminal tail found in all mutant proteins [2, 3] (Fig. 1). The mutant-specific C-terminal lacks the KDEL ER-retention signal and contains an abundance of positively charged amino acids. Since $C A L R$ mutations are found recurrently in approximately $30 \%$ of patients with ET and PMF and are mutually exclusive to $J A K 2$ and $M P L$ mutations, they are believed to play a causal role in MPN development presumably via activation of the MPL and JAK2 signaling pathway.
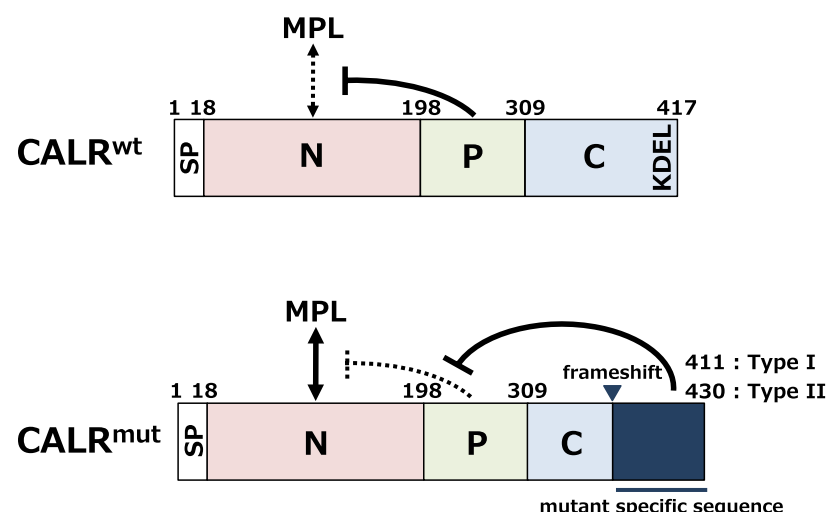

Fig. 1 Domain structure of wild-type and mutant CALR. Wildtype CALR $\left(\mathrm{CALR}^{\mathrm{wt}}\right.$ ) consists of a signal peptide (SP), N-domain $(\mathrm{N})$, P-domain $(\mathrm{P})$, and $\mathrm{C}$-domain $(\mathrm{C})$ with the ER-retention signal KDEL. Because of $+2 /-1$ frameshift mutation, all types of CALR mutant $\left(\mathrm{CALR}^{\mathrm{mut}}\right)$ lose KDEL sequence and gain a novel positively charged amino acid tail. The mutant-specific $\mathrm{C}$-terminal domain interferes with the P-domain and allows interaction between the $\mathrm{N}$-domain and MPL for activation. The numbers refer to the positions of amino acid residues in the amino acid sequence

\section{Phenotypes of animal models}

Mouse models were established and analyzed to examine the involvement of mutant CALR in MPN development. Using a retroviral transduction system, human $C A L R$ with del52 mutation was introduced and expressed in lineage-negative [7] or c-Kit-enriched [8] bone marrow cells, which were then engrafted into lethally irradiated mice. Within 16 weeks after the transplantation, mice developed thrombocytosis with megakaryocytic hyperplasia in the bone marrow [7, 8], phenocopying ET. Upon prolonged observation, mice showed a decrease in blood count associated with myelofibrosis phenotypes such as bone marrow fibrosis and osteosclerosis [7]. In the transplanted mice, red blood cell and white blood cell counts did not increase significantly, suggesting that CALR del52 possesses megakaryocyte lineage-specific oncogenic property. In addition, hematopoietic stem and progenitor cells harboring CALR del52 expanded more in transplanted mice compared to those in control mice and or those harboring $C A L R$ ins5, indicating that $C A L R$ del52 confers growth advantage [7]. In contrast to $C A L R$ del52, transduction of $C A L R$ ins 5 presented weaker phenotypes [7]. Although CALR del52 and ins5 exhibited significantly different phenotypes in mouse, the expression of any of these $C A L R$-mutants led to ET and PMF phenotypes.

More recently, a transgenic mouse model, in which the expression of human $C A L R$ del52 is driven by a $\mathrm{H}-2 \mathrm{~K}$ promoter, has been generated and analyzed [9]. Similar to the retroviral transduction model, these mice showed an increase in platelet count, but not in hemoglobin level or white blood count, with an expansion of mature megakaryocytes in the bone marrow, phenocopying ET. However, these mice did not exhibit the bone marrow fibrosis phenotype. Furthermore, the hematopoietic stem and progenitor cells harboring CALR del52 did not outgrow the wild-type counterparts in a competitive serial transplantation assay [9]. The differential phenotypes observed in these mouse models may be due to the differential strength of the promoters. Nevertheless, these studies demonstrated that $C A L R$ mutation is an oncogenic driver mutation in vivo.

\section{The activation of MPL by mutant CALR}

Although Klampfl et al. reported that expression of mutant CALR induced cytokine-independent proliferation in $\mathrm{Ba} / \mathrm{F} 3$ cells that is associated with an increase in levels of phospho-STAT5 [3], the molecular mechanism underlying this observation was not elucidated. Later, the oncogenic properties of mutant CALR were 
demonstrated by inducing cytokine-independent growth in human UT-7/TPO [10], UT-7 [8], and mouse Ba/F3 $[7,8,11,12]$ and $32 \mathrm{D}[13]$ cells in a manner dependent on the expression of MPL, a thrombopoietin (TPO) receptor. The phenotypes observed in these cell lines recapitulated the formation of endogenous megakaryocytic colonies formed by the cells derived from bone marrow of patients with ET harboring CALR mutations [14]. The MPL-dependent mutant oncogenic property of CALR was then demonstrated in vitro using iPS [10] as well as peripheral blood-origin $\mathrm{CD} 4^{+}[15]$ cells derived from the patients harboring CALR mutation. The lineagenegative mouse bone marrow cells transduced with $C A L R$ mutants showed similar results both in vitro and in vivo [7]. The induction of cytokine-independent growth in the cells was apparently because of the activation of JAK2 and downstream signaling molecules including ERK1/2 and STAT5 (Fig. 2) [7-11, 15]. Conversely, the treatment of cells with JAK inhibitor inhibited the mutant CALRdependent activation of downstream molecules [10]. These data defined CALR mutation as a gain-of-function mutation that induces the JAK2 activation and subsequent cytokine-independent growth. In addition, this provided a rationale to the efficacy of JAK2 inhibitors used to treat the patients with MPNs harboring CALR mutation.

Based on the evidences that mutant CALR exhibited MPL-dependent oncogenic property, physical interaction between mutant CALR and MPL was examined. The interaction between MPL and the mutant, but not the wild-type CALR, has been demonstrated $[8,10]$. Since mutant CALR preferentially interacts with MPL, it was expected that the mutant-specific C-terminus sequence is the binding site for MPL. Interestingly, the N-domain harboring the N-glycan binding property, and not the $\mathrm{C}$-terminus domain, is shown to interact with MPL [10], which is caused by a presumptive structural change induced by the mutation. Domain analysis suggested that the mutant-specific domain blocks the P-domain, which interferes with $\mathrm{N}$-domain binding to MPL in the wild-type CALR, but allows the mutant CALR to preferentially interact with MPL (Fig. 1) [10].

In agreement with the binding of the $\mathrm{N}$-domain of mutant CALR to MPL, amino acid substitutions (D135L/ Y109F) of key residues of the glycan binding site in the $\mathrm{N}$-domain of the CALR mutant abrogated MPL activation [15]. Reciprocally, MPL harboring a substitution in the $\mathrm{N}$-glycosylation site (N117Q) was not activated by mutant CALR [15]. These data strongly suggest that mutant CALR recognizes $\mathrm{N}$-glycosylated MPL in the course of activation. Interestingly, MPL harboring D261A/L265A substitutions that abolish TPO-binding capacity responds to mutant CALR [15], suggesting that the manner of MPL activation differs between mutant CALR and TPO.

Although mutant CALR induces cytokine-independent growth in an MPL-dependent manner, this was not

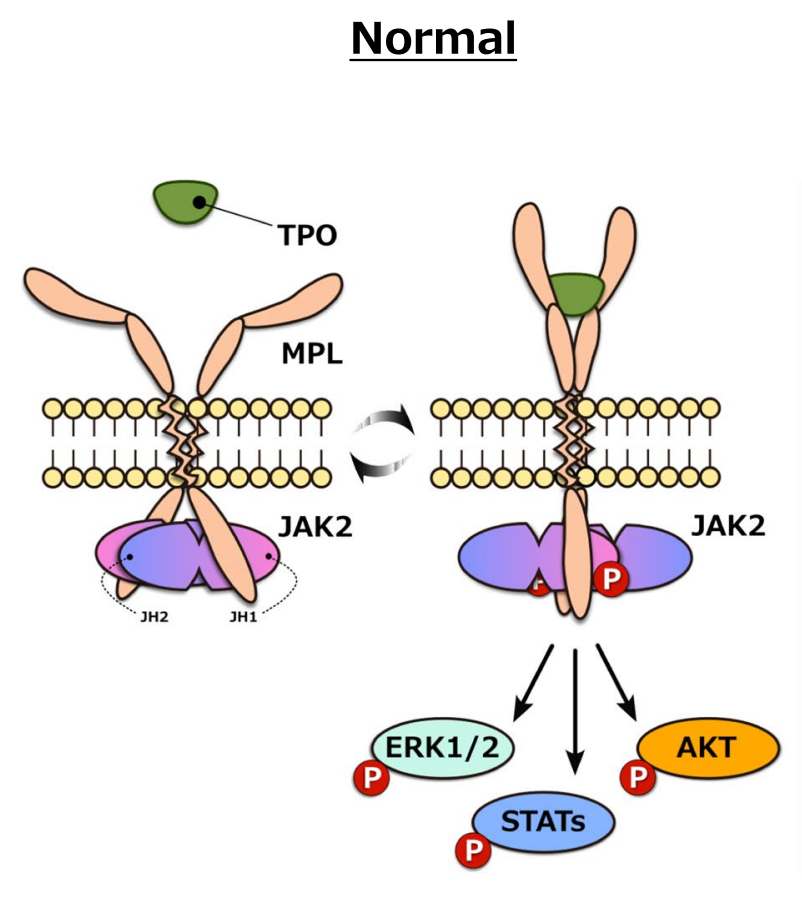

\section{CALR-mutant MPN}
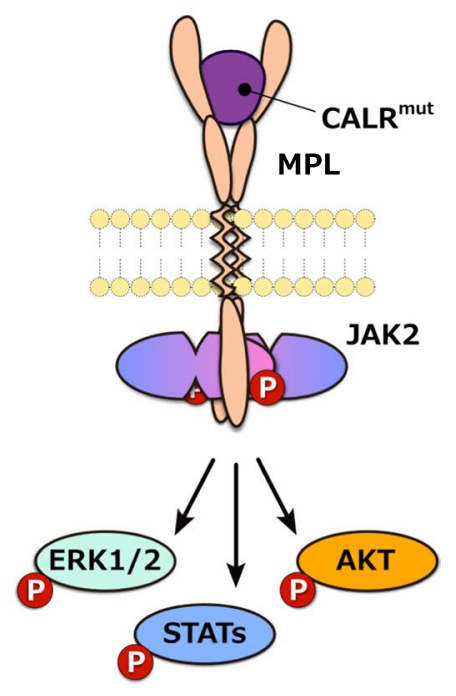

Fig. 2 Constitutive activation of MPL by mutant CALR. Mutant CALR interacts with MPL and activates downstream signaling molecules. Note that mutant CALR-dependent MPL activation may not occur on the extracellular membrane (see text) 
observed with receptors that share structural similarities with MPL such as the erythropoietin receptor (EPOR) [10] and granulocyte-colony stimulating factor receptor (GCSFR) [7, 8]. The preferential activation of MPL by mutant CALR is further demonstrated by the reporter assay, in which the extracellular domain of MPL was shown to be indispensable for mutant CALR-dependent activation of the receptor [15]. Such receptor preference can be explained by the structural difference between MPL and EPOR or GCSFR, which determines the preferential binding of the CALR mutant to MPL, but not to others [8]. However, the CALR mutant interacts with all these receptors [10], which agrees with the weak activation of GCSFR by mutant CALR [15]. These observations suggested that the preferential activation of receptor molecules is determined by the presumptive conformational change induced only in MPL (and in GCSFR at a weaker extent) after binding of mutant CALR [10].

\section{Lack of paracrine capacity in the CALR mutant for the activation of MPL}

CALR was first isolated from the ER and has been used as an ER marker, but it also localizes to the cell surface and is even secreted from the cells. Similarly, cell surface accumulation [10] and secretion [13] of mutant CALR has also been shown. Although this observation suggested that mutant CALR may act as a cell surface-localized ligand or a cytokine, studies showed that it only exhibits autocrine capacity for MPL activation [10, 13]. Why can mutant CALR secreted in the media, not activate MPL on the cell surface? As described in the previous section, mutant CALR is likely to recognize the $\mathrm{N}$-glycosylated MPL for activation. The N-glycosylation and subsequent glycan processing occurs during the receptor maturation in the ER and in the Golgi apparatus, which suggests that mutant CALR only interacts with MPL in certain steps of the maturation, and is thus unable to activate MPL on the cell surface where only the fully mature MPL accumulates. In agreement with this hypothesis, expression of mutant CALR blocked MPL maturation and induced an accumulation of the immature form of MPL in $\mathrm{Ba} / \mathrm{F} 3$ cells [15]. Furthermore, immature MPL was also accumulated on the surface of cells expressing the CALR mutant [15], suggesting that CALR mutant and immature MPL complex may exist in those cells.

Studies showed that oncogenic mutant versions of receptor tyrosine kinases such as FLT3-ITD and c-kit (D814Y) activate downstream targets in the cellular compartments other than cell surface $[16,17]$. Because of the potential involvement of $\mathrm{N}$-glycosylation in mutant CALRdependent MPL activation, a hypothesis for the premature activation of MPL in the ER and/or the Golgi apparatus has been proposed. Although no definitive answer for the location of MPL activation by mutant CALR is reported, evidence showed that mutant, but not wild-type CALR, accumulated in the ER to Golgi intermediate compartment (ERGIC) in mouse embryonic fibroblast cells lacking CALR [15]. Distinctive accumulation of mutant CALR in ERGIC is at least partly, if not completely, due to a lossof ER-retention signal by the frameshift mutation (Fig. 1). However, other evidence suggested that there is no significant difference in the localization of the mutant and wildtype CALR in COS-7 cells [2]. Nevertheless, detailed investigations for identifying the place(s) of MPL activation by mutant CALR is required for understanding the oncogenic event caused by the mutant chaperone.

\section{Conclusions}

Recent findings described here greatly advanced our understanding of the etiology of MPNs induced by CALR mutations. The constitutive activation of the receptor by the mutant molecular chaperone is a novel molecular mechanism for cell transformation. However, the precise molecular mechanism via which mutant CALR interacts and subsequently activates MPL requires further investigation. In addition, the intracellular location where mutant CALR induces MPL and subsequent activation of JAK2 is still ambiguous. These studies may lead to the development of novel therapeutic strategies for the patients with MPN harboring CALR mutation.

Acknowledgements This work was funded in part by the Ministry of Education, Culture, Sports, Science and Technology's Promotion Plan for the Platform of Human Resource Development for Cancer project, Japan Society for the Promotion of Science's KAKENHI Grant \#15K15368, \#16K09859, \#17K16195, \#17H04211, and grants from Takeda Science Foundation, SENSHIN Medical Research Foundation and Japan Leukemia Research Fund. The funders had no role in preparation of the manuscript.

\section{References}

1. Nangalia J, Green TR. The evolving genomic landscape of myeloproliferative neoplasms. Hematology. 2014;2014:287-96.

2. Nangalia J, Massie CE, Baxter EJ, Nice FL, Gundem G, Wedge DC, et al. Somatic CALR mutations in myeloproliferative neoplasms with nonmutated JAK2. N Engl J Med. 2013;369:2391-405.

3. Klampfl T, Gisslinger H, Harutyunyan AS, Nivarthi H, Rumi E, Milosevic JD, et al. Somatic mutations of calreticulin in myeloproliferative neoplasms. N Engl J Med. 2013;369:2379-90.

4. Ostwald TJ, MacLennan DH. Isolation of a high affinity calcium-binding protein from sarcoplasmic reticulum. J Biol Chem. 1974;249:974-9. 
5. Michalak M, Groenendyk J, Szabo E, Gold LI, Opas M. Calreticulin, a multi-process calcium-buffering chaperone of the endoplasmic reticulum. Biochem J. 2009;417:651-66.

6. Gold LI, Eggleton P, Sweetwyne MT, Van Duyn LB, Greives MR, Naylor SM, et al. Calreticulin: non-endoplasmic reticulum functions in physiology and disease. FASEB J. 2010;24:665-83.

7. Marty C, Pecquet C, Nivarthi H, El-Khoury M, Chachoua I, Tulliez M, et al. Calreticulin mutants in mice induce an MPLdependent thrombocytosis with frequent progression to myelofibrosis. Blood. 2016;127:1317-24.

8. Elf S, Abdelfattah NS, Chen E, Perales-Paton J, Rosen EA, Ko A, et al. Mutant calreticulin requires both its mutant C-terminus and the thrombopoietin receptor for oncogenic transformation. Cancer Discov. 2016;6:368-81.

9. Shide K, Kameda T, Yamaji T, Sekine M, Inada N, Kamiunten A, et al. Calreticulin mutant mice develop essential thrombocythemia that is ameliorated by the JAK inhibitor ruxolitinib. Leukemia. 2016;1-9.

10. Araki M, Yang Y, Masubuchi N, Hironaka Y, Takei H, Morishita $\mathrm{S}$, et al. Activation of the thrombopoietin receptor by mutant calreticulin in CALR-mutant myeloproliferative neoplasms. Blood. 2016;127:1307-16.

11. Nivarthi H, Chen D, Cleary C, Kubesova B, Jager R, Bogner $\mathrm{E}$, et al. Thrombopoietin receptor is required for the oncogenic function of CALR mutants. Leukemia. 2016;30:1759-63.
12. Kollmann K, Warsch W, Gonzalez-Arias C, Nice FL, Avezov E, Milburn J, et al. A novel signalling screen demonstrates that CALR mutations activate essential MAPK signalling and facilitate megakaryocyte differentiation. Leukemia. 2017;31:934-44.

13. Han L, Schubert C, Kohler J, Schemionek M, Isfort S, Brummendorf $\mathrm{TH}$, et al. Calreticulin-mutant proteins induce megakaryocytic signaling to transform hematopoietic cells and undergo accelerated degradation and Golgi-mediated secretion. J Hematol Oncol. 2016;9:45.

14. Mondet J, Park JH, Menard A, Marzac C, Carillo S, Pourcelot E, et al. Endogenous megakaryocytic colonies underline association between megakaryocytes and calreticulin mutations in essential thrombocythemia. Haematologica. 2015;100:e176-8.

15. Chachoua I, Pecquet C, El-Khoury M, Nivarthi H, Albu RI, Marty C, et al. Thrombopoietin receptor activation by myeloproliferative neoplasm associated calreticulin mutants. Blood. 2016;127:1325-35.

16. Obata Y, Toyoshima S, Wakamatsu E, Suzuki S, Ogawa S, Esumi $\mathrm{H}$, et al. Oncogenic Kit signals on endolysosomes and endoplasmic reticulum are essential for neoplastic mast cell proliferation. Nat Commun. 2014;5:5715.

17. Choudhary C, Olsen JV, Brandts C, Cox J, Reddy PN, Bohmer FD, et al. Mislocalized activation of oncogenic RTKs switches downstream signaling outcomes. Mol Cell. 2009;36:326-39. 